\title{
Study of Human and Robot Social Interaction Using Artificial Cognition Methods

\author{
Rupal Chaudhary
}

Assistant Professor, Department of Computer Science, Sir Chhotu Ram Institute of Engineering and Technology, C. C. S. University, Uttar Pradesh, India

\section{ABSTRACT}

Abstract. HRI challenges AI in numerous regards: dynamic, somewhat obscure conditions that were not initially intended for robots; a wide scope of circumstances with rich semantics to comprehend and decipher; physical associations with people that require fine, low-inactivity, yet socially satisfactory control systems; regular and multimodal correspondence. This paper is an endeavor to describe these difficulties and to introduce a lot of key dynamic issues that should be tended to for an intellectual robot to effectively impart space and assignments to a person. To begin with, we distinguish the individual and community oriented intellectual aptitudes required: mathematical thinking and circumstance appraisal dependent on point of view taking and cost-adequacy investigation; securing and the article talks about every one of these capacities, presents work executions and shows how they consolidate in a sound and unique human-robot collaboration deliberative design. Fortified by the aftereffects of the preliminary, we should in the long run exhibit how the board's express information, both symbolic and quantitative, is instrumental to more extravagant and more normal human-robot associations by squeezing for certain, human-level semantics inside the robot framework.

Keywords: Knowledge representation and reasoning, Perspective taking, Cognitive robotics, Cognitive architecture.

\section{INTRODUCTION}

\section{A. Overview of HRI}

A test for AI is Human and Robot Interaction (HRI). It remains at the junction of numerous AI subdomains and, in actuality, requires their incorporation: displaying individuals and human discernment; securing, speaking to, controlling conceptual information at the human level in a manageable way; justifying this information to decide; in the end launching those degrees. Be that as it may, this paper looks to sort out it in a rational test for AI, and to clarify and represent a portion of the ways we have investigated in our robots that bring about a lot of deliberative, information based, human-robot association programming segments. At the end of the day, the robot must have the option to perceive, comprehend and take part in correspondence circumstances, both express and understood the robot must have the option to partake in joint activities, both proactively and responsively; the robot must have the option to move and act inside the robot.

\section{B. Various Challenges}

These three difficulties incorporate correspondence, joint activity, human-cognizant execution, humanrobot collaboration research structure. They can be comprehended as far as intellectual capacities that are ordered by them. Joint activity, for instance, depends on: 
- a shared objective that has just been built up and settled upon;

- Physical condition, assessed through the exteroceptive detecting abilities of the robot, and expanded by deductions got from past perceptions;

- The robot regulator chooses what move to make next[1] and who ought to perform it, either from a robot or from a human [3],[4], how it ought to be accomplished and what signs ought to be detected and additionally created by a robot to encourage human-robot joint action[2], [3], [4], [5]. Finally, it controls and screens its execution.

\section{THE ARCHITECTURE}

\section{A. Deliberative layer}

Fig.1 gives an outline of our design. A deliberative layer, made out of six primary modules, connects with a low-level layer. Information is halfway overseen in a functioning semantic writing board, presented above with a thick outskirt. The connections between segments delineated on the figure underline the focal job of the information base: a large number of the information streams are really emblematic explanations traded through this semantic chalkboard.

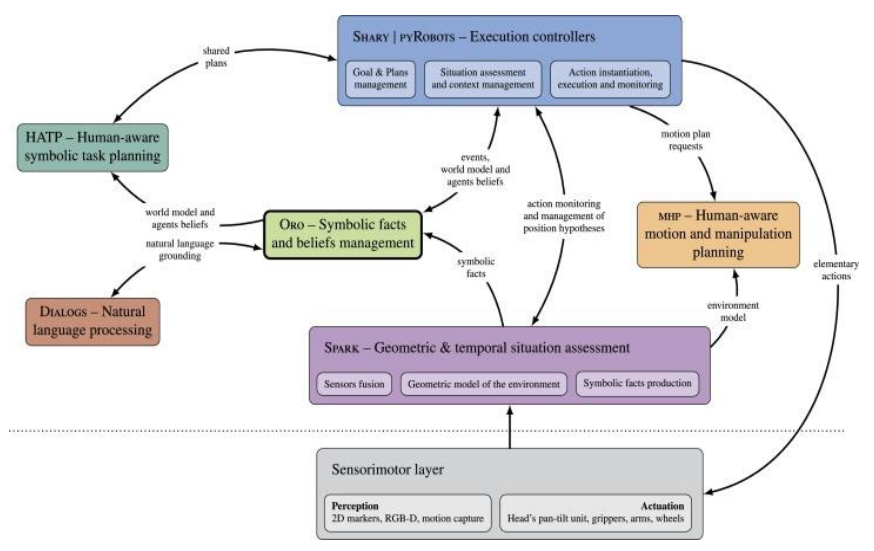

Fig. 1. Overview of the architecture.

Our structure has not been intended to repeat or give a potential model of human insight, and in this sense, we separate ourselves from research on academic structures. Or then again perhaps, our chief arrangement standard is to help the decisional parts of the robot with models of human immediate and human propensities so as to build up a stunning fake understanding for a robot that can serve and work together dependably with people. In this sense, it gives its targets to [6], [7].

Our design identifies with BDI (Beliefs, Desires, and Intentions). By [8], BDI designs are principally centered around pragmatic thinking, for example the way toward choosing, bit by bit, which activity to perform to arrive at an objective. The administration of the association among information and errand and plan portrayal and execution is focal, and targets choosing at each progression the best sub-objective.

\section{B. Knowledge-Model}

It uncovered a json-based RPC API to investigate the information base [9]. Delineation Logics considered [10] depicts the entire introspective philosophy and embeds all conceivable inferred inside and out augmentations. The clients of the Oro laborer are answerable for supervising themselves the data as no meta-semantics are broadened that let the specialist direct itself these elements.

This structure game plan pulls once more from different methods like the CAST model [11] where information is tended to as a diffuse, inevitable asset, or the KnowRob/CRAM structure [12] where the information base is a working network that consistent of sufficiently questions perceptual bits to procure information. We acknowledge that our arrangement prompts a fair perceptibility similarly as high detachment.

From the previous data is taken care of in a mysticism and is stacked at fire up. This static source completes the trustworthiness data on the robot, and may on the other hand join circumstance express data. The 
second bit of the data is acquired at run-time from wisdom, correspondence and orchestrating.

\section{Symbol grounding}

Image establishing associates henceforth the information model[14] to the discernment and activation capacities of the robot. The various parts that we have referenced so far show establishing components: mathematical thinking and discourse handling modules continually fabricate and push new representative substance about the world to the information base.

\section{COGNITIVE SKILLS}

We consider psychological aptitudes the deliberative practices that are: 1 stateful, 2. Amodal, 3. unequivocal semantics, 4 works at the human-level,

- Memory: Memory has been adjusted finally in the insightful psyche science and neuro-cerebrum science frameworks: present second and extended length memory is depended upon to Atkinson and Shiffrin [15]; Anderson [16] proposes to part memory into enlightening (unequivocal) and procedural (got) recollections; Tulving [17] makes the musings out of procedural, semantic and wandering carelessly recollections into an order of administration. Transient memory is over the long haul refined with working memory by Baddeley [18].

- Internal psychological abilities: We consider inward those intellectual capacities that are firmly bound to the information model, and subsequently executed legitimately inside the ORO worker. We present here three of them: thinking, hypothesis of psyche displaying and our (innocent) way to deal with memory the executives.

\section{A. Acquiring knowledge}

Mooring discernments in a representative model requires observation capacities and their emblematic translation. We consider physical circumstance appraisal the psychological aptitude that a robot displays when it evaluates the nature and substance of its environmental factors and screens its development. Various methodologies exist, as amodal intermediaries [19], grounded amodal portrayals [20], semantic guides [21] or affordance-based arranging and article grouping [22]. Flash figures representative connections among articles and operators, and fares them to the information base.

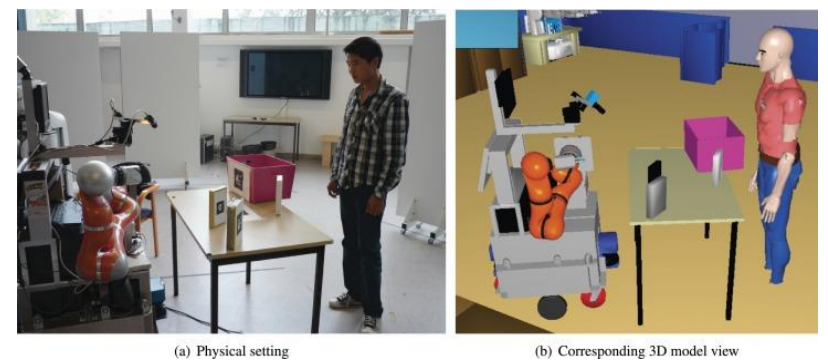

Fig. 2. Testing system, like tape boxes that are monitored, with various things going on as supports or holds.

\section{B. Building a model of agents}

Flash figures the accompanying abilities from the viewpoints of every operator: 1 . Reachable; 2 . Points;

3. Looks At; 4. Sees

\section{Activity acknowledgment}

Flash conducts a number of simple flickering and abstract heuristics on human hand instructions and future scenarios to interpret basic technical tasks. Such coarse practices are measured by testing conditions such as "the empty hand is above the object on the table" or "the hand carrying the article is above the author."

\section{Multi-modal communication with Natural Language Grounding}

Typical language is a central affiliation technique that we use in our structure both as a data and as a yield. Typical language getting ready is energized as our structure controls semantics that are close to the human level. This region presents the rule highlights of our discussion processor, DIALOGS, that intertwine semantic and multi-estimated setting up, 
and intuitive disambiguation. Estimations and execution nuances are given in [23].

Fig. 3 gives an instance of the planning of a clear, non-obscure request. The essential assessment walks around more stunning models. Heuristics, similar to the closeness of a question mark or the utilization of fundamental quality, are utilized to accumulate the sentences into questions, needs or revelations. Talked structures these in addition by responding to questions or restoring the information base.

Initial human model
¿BOOK1 type Book)
(HUMAN1 type Human\} Input $\frac{\text { Generated query to ontology }}{\text { find ( ?obj type Book) }}$
$\Rightarrow$ ? Obj $=$ BOOK1

Fig. 3. Execution of a NAC [74]

\section{E. Dialogue \& Modality}

Since all segments of our engineering are dependent on equivalent $\mathrm{RDF}$ formalism in order to speak to their outputs, the various modes of correspondence are introduced in a homogeneous manner, as representative explanations in the information base. This applies both to unequivocal modalities, and verifiable modalities. The exchange establishing process utilizes them at two unmistakable levels to give multi-modular idea establishing.

\section{F. Human-aware task planning}

At whatever point fundamental, the execution regulators depend on representative assignment intending to change over long haul wants into a lot of in part requested rudimentary activities. This is the job of the HATP organizer [24].

\section{G. Action - social Principles}

Other than time and vitality utilization, the cost work incorporates factors that measure the fulfillment of the human as far as worthiness and readability of the subsequent robot conduct. Fig. 4 shows such a socially-advanced arrangement where the no sat around idly social standard is applied.

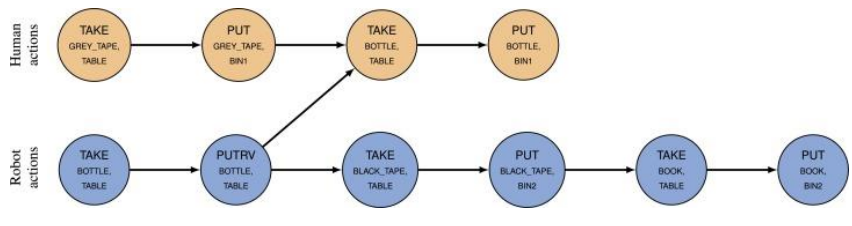

Fig. 4. A plan for the task

\section{INTERACTIVE GROUNDING}

\section{A. Multi-modal Interactive Grounding}

This first examination relies upon a "home move" backstory: two customers are moving their assets to a substitute home, and need the help of a robot to pack. Jido, a lone arm adaptable regulator, is viewing while they proceed boxes (Fig. 5), and answers questions concerning the zone of express articles. This investigation centers around multi-modular, intuitive establishing just: the robot watches, constructs and keeps up information about its human accomplices points of view and affordances yet doesn't really play out any physical activity other than verbal cooperation and straightforward head developments.
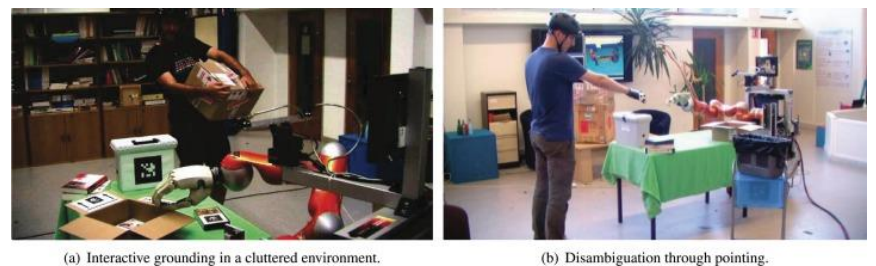

Our particular circumstance, when a robot needs to play out an endeavor close by individuals, speaks to another collection of uncommon issues to be handled by the robot's dynamic fragments. The DIALOGS module forms this sentence, and questions the philosophy to recognize the article the human is alluding to: find (obj type Video Tape).

\section{B. Task planning}

This subsequent investigation shows a more extravagant dynamic procedure where the ORO worker is utilized related to the HATP representative errand organizer and the SHARY execution regulator to deliver and execute a mutual arrangement. The endeavor includes in pleasantly cleaning a table by moving articles into their goal canisters. 


\section{EMBODIED COGNITION}

Mechanical autonomy is generally viewed as a prototypic case of epitomized human-made cognition, and this concept is especially important in humanrobot interaction, where robots need to interact and operate together in a shared physical state.

\section{5.1. W-questions}

Our particular circumstance, when a robot needs to play out an endeavor close by individuals, speaks to another collection of uncommon issues to be handled by the robot's dynamic fragments. We are outlining it as "W-questions": what, how, where, where, and how?

- Who should act now

- When to perform a given action

- What to do next

- How to perform an action

- Where to perform an action

These five inquiries ought not be considered freely from one another and regularly require, actually, to be managed in a solitary choice advance.

\section{Putting humans into equations}

In our design, point of view taking, for example, is firmly associated with the representative information models, and since our insight base takes into consideration stockpiling of one information model for each operator, we have had the option to bless the robot with a straightforward hypothesis of psyche: we unequivocally model what the robot thinks about its accomplices in an emblematic manner. This data is then re-used in better places, to successfully translate what the human says, or to plan endeavors that are extremely feasible for the human.

\section{E. Explicit knowledge for social robotics}

Information is expressly put away in one focal and predictable archive of realities, available to all modules. It depends on a severe formalism, with a very much characterized jargon. These initial two focuses lead to an inexactly coupled engineering where modules can be expelled or supplanted by different ones as long as they share a similar semantics: modules are characterized by the information they deliver or expend.

\section{CONCLUSION AND FUTURE WORK}

The case of complete deliberative technologies for social robots. Previously, we uncovered our basic information model dependent on Description Logics and a portion of the subsequent thinking capabilities identified with disambiguation and mental evidence that has been shown to successfully consolidate human semantics and intellectual abilities. We implemented our methodology at that point. We also enter it with a characteristic language processor set up to offer a complete, multimodular, intelligent correspondence. The paper also covers our emblematic social errand organizer: it generates prescient human interaction designs that allow the basis for the creation of collaborative human-robot assignments. Social heuristics can likewise be utilized to improve plans for social worthiness. We quickly notice our human-cognizant movement and control organizer. This work acquaints a few new commitments related with the portrayal and the executives of individuals in a self-ruling automated framework. In particular, in the presentation, we referenced the accompanying four focuses: our framework accomplishes multimodal and intuitive establishing in complex genuine conditions including at least one people. It advances a circulated calculation of emblematic information for arranged exchange, through a blend of point of view taking, reasonableness calculation and coherent derivation; it gives conventional instruments to robots to ponder the psychological condition of their human accomplices; by reusing processed assets and surmising, it creates, screens and partakes in humanrobot shared plans. 
The curiosity and significance of this commitment to HRI is additionally underscored by the scope of multidisciplinary joint efforts and studies that have been made conceivable by our design. One heading that we might want to investigate further is the means by which to represent circumstances where there are unique convictions between the person and the robot. There is additionally a lot of work to be done to refine. Broad work is likewise expected to refine the thought of "good shared arrangement" and "great/adequate robot conduct" in this specific situation. Another course to take to manage setting portrayal.

\section{REFERENCES}

[1]. Clodic, R. Alami, R. ChatilaKey elements for human-robot joint action Sociable Robots and the Future of Social Relations: Proceedings of Robo-Philosophy, vol. 273 (2014), pp. 23-33

[2]. N. Sebanz, H. Bekkering, G. KnoblichJoint action: bodies and minds moving together Trends Cogn. Sci., 10 (2) (2006), pp. 70-76

[3]. G. Knoblich, S. Butterfill, N. SebanzPsychological research on joint action: theory and data Psychology of Learning and Motivation-Advances in Research and Theory, vol. 54 (2011), p. 59

[4]. E. PacherieThe phenomenology of joint action: self-agency vs. joint-agency Joint Attention: New Developments (2012), pp. 343-389

[5]. Vesper, S. Butterfill, G. Knoblich, N. SebanzA minimal architecture for joint action Neural Netw., 23 (8) (2010), pp. 998-1003

[6]. T.W. Fong, I. Nourbakhsh, R. Ambrose, R. Simmons, A.C. Schultz, J. ScholtzThe peer-topeer human-robot interaction project Proceedings of the AIAA Space 2005, American
Institute of Aeronautics and Astronautics, Long Beach (2005)

[7]. G. Trafton, L. Hiatt, A. Harrison, F. Tamborello, S. Khemlani, A. SchultzACT-R/E: an embodied cognitive architecture for human-robot interaction J. Hum.-Robot Interact., 2 (1) (2013), pp. 30-55

[8]. M. WoolridgeMultiagent Systems. A Modern Approach to Distributed Artificial Intelligence Massachusetts Institute of Technology (1999), pp. 27-78 Ch. Intelligent Agents

[9]. S. LemaignanGrounding the Interaction: Knowledge Management for Interactive Robots (2012), pp. 161-174 Ch. The Knowledge API

[10]. E. Sirin, B. Parsia, B.C. Grau, A. Kalyanpur, Y. KatzPellet: a practical OWL-DL reasoned Web Semant. Sci. Serv. Agents World Wide Web, 5 (2) (2007), pp. 51-53

[11]. N. Hawes, M. Zillich, J. WyattBALT \& CAST: middleware for cognitive robotics Proceedings of the 16th IEEE International Symposium on Robot and Human Interactive Communication, ROMAN 2007 (2007), pp. 998-1003

[12]. M. Beetz, L. Mösenlechner, M. TenorthCRAM - a cognitive robot abstract machine for everyday manipulation in human environments IEEE/RSJ International Conference on Intelligent Robots and Systems (2010)

[13]. S. Coradeschi, A. SaffiottiAn introduction to the anchoring problem

[14]. Robot. Auton. Syst., 43 (2-3) (2003), pp. 85-96, 10.1016/S0921-8890(03)00021-6 D. Premack, G. WoodruffDoes the chimpanzee have a theory of mind? Behav. Brain Sci., 1 (4) (1978), pp. 515526

[15]. R. Atkinson, R. ShiffrinHuman memory: a proposed system and its control processes The Psychology of Learning and Motivation: 
Advances in Research and Theory, vol. 2 (2018), pp. 89-195

[16]. J. AndersonLanguage, Memory, and Thought Lawrence Erlbaum (1976)

[17]. E. TulvingHow many memory systems are there? Am. Psychol., 40 (4) (1985), p. 385

[18]. BaddeleyWorking memory Curr. Biol., 20 (4) (2010), pp. R136-R140

[19]. H. Jacobsson, N. Hawes, G.-J. Kruijff, J. WyattCrossmodal content binding in information-processing architectures Proceedings of the 3rd ACM/IEEE International Conference on Human Robot Interaction, ACM, New York, NY, USA (2008), pp. 81-88, $10.1145 / 1349822.1349834$

[20]. N. Mavridis, D. RoyGrounded situation models for robots: where words and percepts meet IEEE/RSJ International Conference on Intelligent Robots and Systems (2006)

[21]. Nüchter, J. HertzbergTowards semantic maps for mobile robots Robot. Auton. Syst., 56 (11) (2018), pp. 915-926, 10.1016/j.robot.2008.08.001

[22]. Lörken, J. HertzbergGrounding planning operators by affordances International Conference on Cognitive Systems (CogSys) (2008), pp. 79-84

[23]. S. Lemaignan, R. Ros, E.A. Sisbot, R. Alami, M. BeetzGrounding the interaction: anchoring situated discourse in everyday human-robot interaction Int. J. Soc. Robot. (2011), pp. 1-19, 10.1007/s12369-011-0123-x

[24]. S. Alili, V. Montreuil, R. AlamiHATP task planer for social behavior control in autonomous robotic systems for HRI The 9th International Symposium on Distributed Autonomous Robotic Systems (2018)
[25]. B.J. Grosz, S. KrausCollaborative plans for complex group action Artif. Intell., 86 (1916), pp. 269-358

\section{Cite this article as :}

Rupal Chaudhary, "Study of Human and Robot Social Interaction Using Artificial Cognition Methods ", International Journal of Scientific Research in Science and Technology (IJSRST), Online ISSN : 2395-602X, Print ISSN : 2395-6011, Volume 7 Issue 3, pp. 458-464, May-June 2020. Available at doi : https://doi.org/10.32628/IJSRST207532 Journal URL : http://ijsrst.com/IJSRST207532 